\title{
Title: Long-term health related quality of life following Uterine Fibroid Embolization 1 in a predominantly black African population: A retrospective Cohort study
}

\author{
Marleen Temmerman \\ Aga Khan University \\ Timona Obura \\ Aga Khan University \\ Adelaide Lusambili \\ Aga Khan University \\ Michael Kioko ( $\square$ mickiks@gmail.com ) \\ Aga Khan University
}

\section{Research Article}

Keywords: Uterine Fibroid Embolization, Uterine Fibroid Symptom and Quality of Life questionnaire, Health-related Quality of Life (HRQOL)

Posted Date: March 5th, 2021

DOI: https://doi.org/10.21203/rs.3.rs-267647/v1

License: (9) This work is licensed under a Creative Commons Attribution 4.0 International License.

Read Full License 
1 Title: Long-term health related quality of life following Uterine Fibroid Embolization

2 in a predominantly black African population: A retrospective Cohort study

3 AUTHORS

4 Dr. Michael Kioko (Corresponding author),

5 Department of Obstetrics and Gynaecology

6 Aga Khan University Hospital, Nairobi

7 Kenya

8 mickiks@gmail.com

10 Professor Marleen Temmerman,

11 Department of Obstetrics and Gynaecology

12 Center of Excellence in Women and Child Health Aga Khan University Nairobi

13 Kenya

14 Marleen.temmerman@aku.edu

15

16 Dr. Timona Obura,

17 Department of Obstetrics and Gynaecology

18 Aga Khan University Hospital Nairobi

19 Kenya

20 timona.obura@aku.edu

22 Dr. Adelaide Lusambili,

23 Aga Khan University Hospital Nairobi

24 Kenya

$25 \quad$ Adelaide.lusambili@aku.edu

AFFILIATION OF THE AUTHORS

29 Dr. Michael Muthoka (MM), Dr. Timona Obura (TO) and Prof. Marleen Temmerman (MT) are in 30 the Department of Obstetrics and Gynecology, Aga Khan University, Nairobi. Prof Marleen

31 Temmerman is also the Director of the Center of Excellence in Women and Child Health, Aga 32 Khan University, Nairobi where Dr. Adelaide Lusambili (AL) is based. 
Background: Uterine Fibroid Embolization (UFE) is one of the effective options available for treatment of symptomatic uterine fibroids with documented improvement in the quality of life and reduction in symptoms. Most of the studies have demonstrated an improvement in the quality of life over a short to intermediate period. We carried out this study to assess the long-term quality of life following uterine fibroid embolization in a predominantly black population. There are no identified studies locally or in Africa addressing this issue whereas studies done elsewhere indicate a probable increased fibroid disease burden among black women.

Methods: This was a retrospective single cohort study that recruited patients who had UFE between 2009-2014. Study participants were sampled consecutively, invited to the study and asked to fill an online general demographic tool and the UFS-QOL. Wilcoxon signed rank tests were done to test for statistical significance between HRQOL scores. Pearson correlation analysis was carried out to evaluate possible association between various independent factors and specific outcomes of interest such as quality of life and symptom severity score.

Results: Data was obtained from 77 participants. The median duration after the initial UFE was eight years. The median health related quality of life was 88.6 (62.9-98.3). This was statistically significant from baseline median scores of similar studies. The median symptom severity score decreased from a baseline score of 54.7 (43.8-65.6) to 21.9 (6.3-42.2). This decrease was statistically significant ( $p$-value $<0.001$ ). Twenty-four participants $(31.1 \%$ ) reported follow up fibroid treatments after the initial UFE procedure. Eleven participants (14.3\%) reported major repeat procedures (myomectomy, hysterectomy and UFE) .Seventeen participants (22\%) reported a pregnancy after the UFE procedure with ten participants (13\%) reporting to have had children after the UFE procedure.

Conclusion: UFE results in clinically sustained improvement in health related quality of life and symptom control among patients with uterine fibroids.

Key Words: Uterine Fibroid Embolization; Uterine Fibroid Symptom and Quality of Life questionnaire; Health-related Quality of Life (HRQOL)

\section{BACKGROUND}

Uterine fibroids are the commonest benign pelvic neoplasms among women of reproductive age leading to significant healthcare burden in both direct and indirect costs $[1,2]$. The exact 
worldwide prevalence and incidence is unknown. In 2013, the Lancet estimated that at least 171 million women were affected [3]. Various regional studies estimate the prevalence of symptomatic fibroids at between $11 \%$ to $35 \%[2,4]$. Up to $50 \%$ of women are asymptomatic but have sonographic evidence of uterine fibroids[2]. Racial differences have also been described with regards to the symptomatology, age of onset, number and size of fibroids, rates of hysterectomy due to fibroids and regression following menopause. Compared to white women, research shows that black women have an increased risk of fibroids, are younger at diagnosis, are more symptomatic, have larger and more numerous fibroids and are more likely to require surgery for fibroids[2, 5-10]. Further evidence illustrates that the growth rate of fibroids also reduced with age among whites but not for blacks[11].

Uterine fibroids present with a wide array of symptoms that depend on the number, size and location of fibroids. Menstrual disturbances predominate in up to two-thirds of patients with symptomatic fibroids [12]. Other common symptoms include bulk related symptoms and pelvic pain[12]. These symptoms can lead to decreased quality of life hence needing treatment for uterine fibroids[13].

Uterine Fibroid Embolization (UFE) is a minimally invasive technique which has been in use for management of uterine fibroids for roughly two decades now[14]. It involves delivery of embolic material such as gel foam particles, polyvinyl alcohol and microspheres under fluoroscopic guidance leading to occlusion of blood flow to fibroids. This results in ischemia and hyaline degeneration of the fibroids with attendant shrinkage and fibroid related symptom control or resolution in up to $90 \%$ of the patients $[15,16]$. It is currently a standard of treatment in many high-income countries. Although it is used in Africa, it is limited due to unavailability of expertise and cost implications in resource-constrained settings. Several studies have documented its effectiveness and safety in management of symptomatic fibroids with a low rate of major adverse events and high patient satisfaction.[17]. Patients undergoing fibroid embolization have demonstrated a significant improvement in the quality of life in the short term and intermediate follow up $[15,18,19]$. Other benefits of UFE that have been described include short hospital stay and swift return to daily activities $[17,20]$. Various studies have documented the long-term follow up of the patients post UFE in an effort to assess the sustenance of symptom control. The key main areas assessed included the proportion of patients with symptom control, the rates of hysterectomy and other follow up procedures due to persistent or recurrent symptoms. The duration of follow up for these studies was $5-7$ years. Those with symptom control varied from 
$53 \%$ to $82 \%$ while the rates of hysterectomy varied from $5-28 \%[21-25]$. We carried out this study to assess the long-term quality of life following uterine fibroid embolization at Aga Khan University hospital, Nairobi using a validated disease specific questionnaire. There are no identified local studies and studies in Africa addressing this issue whereas studies done elsewhere indicate a probable increased fibroid disease burden among black women. The risk of recurrence of fibroid symptoms and the subsequent reduction in the related quality of life increases with time. Hence, there was need to assess the long-term efficacy of UFE using a validated fibroid specific questionnaire in our setting. We hence carried out this study to generate and increase knowledge of UFE as alternative to fibroid in our local setting and in Africa as a whole.

\section{METHODS}

This retrospective single cohort study was undertaken at Aga Khan University Nairobi between July 2019 and March 2020. The primary objective of this study was to determine the long-term health related quality of life following Uterine Fibroid Embolization at Aga Khan University Hospital Nairobi, a private tertiary hospital at Nairobi, Kenya. Secondary objectives included assessment of the symptom severity among the participants, pregnancy rates and follow up treatments after the initial UFE procedure.

The study used the Uterine Fibroid Symptom and Quality of Life questionnaire (UFS-QOL) to collect the data on ongoing symptoms and the quality of life of the participants. The study also obtained various demographic data of the participants. The UFS-QOL questionnaire is a validated disease specific questionnaire for assessment of symptoms and quality of life due to uterine fibroids. The UFS-QOL has two scales: the symptom severity scale (SSS) and health related quality of life scale (HRQOL). The SSS consists of eight questions scored on 5-point Likert scale. The HRQOL on the other hand has 29 questions with six subscales related to quality of life such as concern, assessment of control, tiredness/mood, activities, being self-conscious and effect on sexual function. Like the symptom severity scale, the health related quality of life scale was scored on 5-point Likert score. The sum of points of both scales was obtained and transformed as described in the UFS-QOL[26]. The higher the score on the SSS, the more symptomatic a patient was. The higher the score on the HRQOL Scale, the better the quality of life. Hence, the relationship between these two scales was inverse. The sample size was calculated using the formula for estimating sample size for Likert scale described by Jinwoo et al [27]. 
125 Following approval from the Aga Khan University ethics and research committee the study 126 investigators enrolled patients who had UFE at AKUHN between 2009 and 2014. Study 127 investigators and research then contacted potential participants via email using the information 128 obtained from the hospital records. Each potential participant was emailed a personalized letter 129 on the survey. The letter introduced the survey by outlining the instructions concerning 130 participation and their purpose in taking part in the survey. The invitation email also outlined 131 issues such as confidentiality and benefits of taking part in the survey. In addition, the letter also 132 had contact information of the study investigators such as email and telephone number for clarification of any issues that may arise. Within a week of emailing the letter of introduction, the research assistants called and sent the potential participants a link to the survey via either email, text message or WhatsApp. Clicking on this link redirected the potential participants to the Survey Monkey website specific to the study that had the data collection tools (informed consent, the data demographic tool and the questionnaire). The participants accessed these online data collection tools via any computer or smartphone with internet access.

The participants were required to read and accept the informed consent before proceeding with the study. Any clarification concerning participating in the study was readily available from the study investigators via email, telephone call or text message. The participants were able to access the questionnaire only after reading and accepting the informed consent. The study investigators sent reminder emails to the potential participants who failed to respond to initial prompts. The frequency of the reminders as described in this protocol were every three to four days for the 1st four weeks, then weekly for another four weeks, then once every two weeks for four weeks. If the response rate was still poor, the study investigators/ research assistants made a follow up telephone call to the non-responders. This frequency of reminders was arbitrary and aimed to maximize the response rate.

149 The collected data were analyzed using IBM SPSS statistics version 23 and descriptive statistics 150 tabulated. The scores from the UFS-QOL questionnaire were transformed as described in the original validation study of the questionnaire(38). The data was skewed hence continuous variables were presented as median and interquartile range. Categorical variables were presented as frequencies and percentages. Evaluation for statistical significance between the current HRQOL 154 score and baseline scores in similar studies was done using one sample Wilcoxon signed rank test 155 (19, 20, 28). To evaluate for the statistical significance between baseline and current symptom 156 severity, related samples Wilcoxon signed rank test was employed. Pearson correlation analysis 
157 was carried out to evaluate possible association between various independent factors and specific 158 outcomes of interest such as quality of life and symptom severity score. The strength and direction 159 of association was deemed significant with a p-value of less than 0.05 . In order to further examine 160 associations using a linear regression model between various independent factors and outcomes

161 of interest such as symptom severity and quality of life, preliminary analysis was done to ensure 162 that there was no violation of the assumptions of linearity on the various identified predictor 163 factors. The continuous data was log transformed and the various factors were then modelled 164 into the linear regression model. A p-value cut-off of 0.05 was used to identify the significant 165 associated factors of symptom severity and quality of life.

\section{RESULTS}

167 Consecutive sampling was done until the minimum sample size was obtained. We got 77 168 responses due to the nature of data collection method where some participants who initially were 169 unreachable filled the questionnaire later hence increasing the number of responses. Various 170 demographic characteristics such as baseline age, uterine volume, dominant fibroid volume, 171 symptom score, fibroid number, fertility goal and anemia status were extracted from the AKUHN 172 UFE database. Other demographic data values were collected prospectively as shown in the 173 descriptive tables 1 and 2. Figure 1 below summarizes the participant recruitment process. 


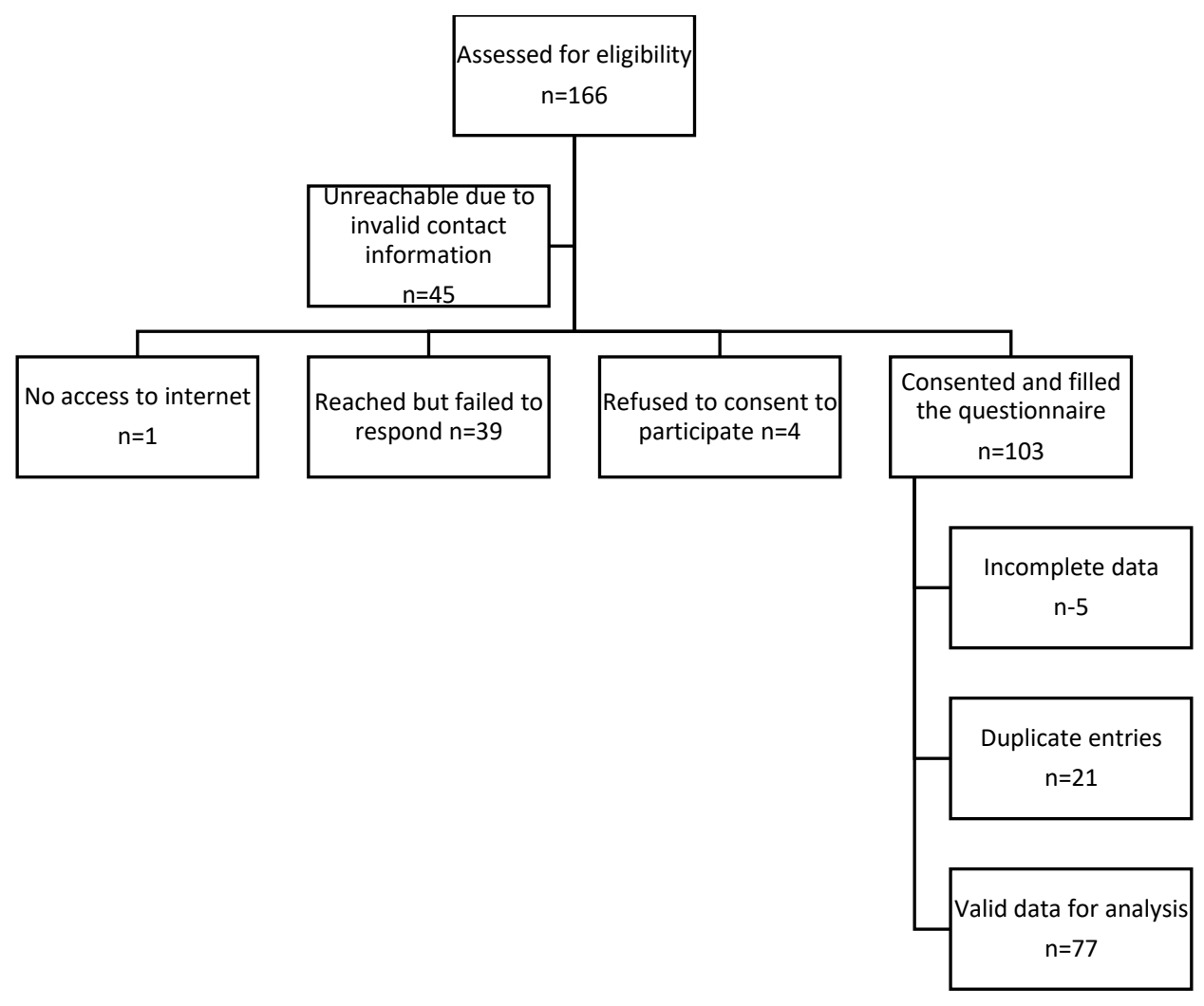

175 Figure 1: Participant enrollment flow to the UFE Study

$176 \quad 3.1$ Socio-economic and clinical characteristics of the participants

177 The median age of the study participants was estimated to be 50 years. The median age at initial

178 UFE procedure was 43 years. The median duration after the UFE procedure was eight years with

179 an interquartile range of $6-9$ years. Majority of the participants identified as of black race and

180 had achieved a tertiary level of education. Thirty-four participants (45\%) reported a family history

181 of fibroids. Twenty-four participants (31.1\%) reported follow up fibroid treatments after the initial

182 UFE procedure. Eleven participants (14.3\%) reported major repeat procedures (myomectomy,

183 hysterectomy and UFE) while thirteen participants (16.9\%) reported various medications to

184 control ongoing fibroid symptoms. Forty out of fifty-five participants $(72.7 \%)$ were reportedly

185 anemic at the initial UFE procedure. The median uterine and dominant fibroid volume as

186 determined from magnetic resonance imaging was using the ellipsoid formula was $632 \mathrm{mls}$ and

$187103 \mathrm{mls}$ respectively. Twenty-one out of forty nine (42.9\%) participants had more than twenty

188 fibroids on MRI scan with 33 participants (67.3\%) having 10 fibroids or more. Thirty out of fifty-

189 five participants (54.5\%) indicated that they had fertility ambitions before the initial UFE

190 procedure. Only seventeen participants (22\%) reported having become pregnant since the UFE 
191 procedure. Ten of these pregnancies (58.8\%) ended up with a live birth while seven (41.2\%) 192 ended in a miscarriage. Sixty-two (80.5\%) of the participants implied satisfaction with UFE by 193 indicating they would recommend the procedure to a family member or friend. The following 194 tables 1 and 2 summarize the descriptive data of the participants.

195 Table 1: Descriptive statistics of study participants at recruitment

\begin{tabular}{|c|c|c|}
\hline & & $\mathbf{N}(\%)$ \\
\hline Age at recruitment (Median, IQR) & & $\begin{array}{l}50(47- \\
53)\end{array}$ \\
\hline Years after UFE Procedure(Median, IQR) & & $8(6-9)$ \\
\hline \multirow[t]{3}{*}{ Race } & Black & $73(94.8)$ \\
\hline & Asian & $1(1.3)$ \\
\hline & Other & $3(3.9)$ \\
\hline \multirow[t]{2}{*}{ Education } & Tertiary Level & $76(98.7)$ \\
\hline & Secondary Level & $1(1.3)$ \\
\hline \multirow[t]{4}{*}{ Marital Status } & Single & $37(48.1)$ \\
\hline & Married & $38(49.4)$ \\
\hline & Separated & $1(1.3)$ \\
\hline & Divorced & $1(1.3)$ \\
\hline Family History & Yes & $34(44.2)$ \\
\hline Additional Procedures & & 24(31.2) \\
\hline \multirow[t]{3}{*}{ Major Additional Procedures } & Myomectomy & $9(11.7)$ \\
\hline & Hysterectomy & $1(1.3)$ \\
\hline & UFE & $1(1.3)$ \\
\hline \multirow[t]{3}{*}{ Minor Additional Procedures } & $\begin{array}{l}\text { Medication to control } \\
\text { bleeding }\end{array}$ & $7(9.1)$ \\
\hline & Hematinic & $3(3.9)$ \\
\hline & Analgesics & $3(3.9)$ \\
\hline Pregnancy after the UFE Procedure & & $17(22.1)$ \\
\hline Children after the UFE Procedure & & $10(13)$ \\
\hline
\end{tabular}


197 Table 2: Descriptive statistics of study participants*

\begin{tabular}{|c|c|c|}
\hline Variable & & N (\%) \\
\hline Baseline age $(n=55)$ (Median, IQR) & & $43(39,45)$ \\
\hline Uterine Volume $(n=52)($ Median, IQR) & & $632.5(439.2,1240)$ \\
\hline $\begin{array}{l}\text { Dominant Fibroid volume }(n=52) \text { (Median, } \\
\text { IQR) }\end{array}$ & & $103.5(56.25,235.5)$ \\
\hline $\begin{array}{l}\text { Baseline symptom score }(n=52) \text { (Median, } \\
\text { IQR) }\end{array}$ & & $54.7(43.8,65.6)$ \\
\hline Fibroid number $(n=49)$ & Less than 5 & $8(16.3)$ \\
\hline & 5 to 9 & $8(16.3)$ \\
\hline & 10 to 14 & $8(16.3)$ \\
\hline & 15 to 20 & $4(8.2)$ \\
\hline & More than 20 & $21(42.9)$ \\
\hline Fertility goal $(n=55)$ & Yes & $30(54.5)$ \\
\hline & No & $24(43.6)$ \\
\hline & Not sure & $1(1.8)$ \\
\hline Anemia $(n=55)$ & Yes & $40(72.7)$ \\
\hline & No & $15(27.3)$ \\
\hline
\end{tabular}

*Baseline - before undergoing the uterine fibroid embolization procedure

\subsection{Quality of life outcomes}

201 The median health related quality of life score among the participants was $88.9(62.9,98.3)$ as

202 shown in figure 2 below. Statistical analysis using the one sample Wilcoxon signed rank test at 203 a significant value of 0.05 indicated was statistically significant different from the study findings 204 in similar studies both at baseline and in the follow up post intervention[18, 19, 28] as shown in 205 table 4 below. Our study findings indicated a much better quality of life from the baseline results 206 of these studies. The mean and median HRQOL subscale domains scores as shown in table 3 
207 below. Sexual function subscale domain was leading with a score of 100 while the self-conscious

208 subscale domain was lagging behind with a score of 83.3 as shown in figure 3 below. Health

209 related quality of life was inversely related to symptom severity as shown in figure 5.

210 Table 3: Health related quality of life and subdomain scores

\begin{tabular}{lll} 
Variable $(\mathbf{n}=\mathbf{7 7})$ & Mean(SD) & Median(IQR) \\
\hline HRQOL Score & $\mathbf{7 7 . 3 ( 2 6 . 3 )}$ & $\mathbf{8 8 . 9 ( 6 6 . 9 - 9 8 . 3 )}$ \\
Subdomain Scores & & \\
Concern & $75.3(31.1)$ & $90.0(52.5-100.0)$ \\
Activities & $79.6(27.3)$ & $92.9(73.2-100.0)$ \\
Energy/Mood & $77.6(27.3)$ & $89.3(62.5-100.0)$ \\
Control & $77.0(27.6)$ & $90.0(52.5-100.0)$ \\
Self-Conscious & $75.3(27.2)$ & $83.3(58.3-100.0)$ \\
Sexual Function & $77.1(31.1)$ & $100.0(62.5-100.0)$ \\
\hline
\end{tabular}

211

212 Table 4: Comparison of health related quality of life outcomes with baseline scores in

213 similar studies

\begin{tabular}{llllll} 
Author & Year & N & average age & Baseline HRQOL scores & $\begin{array}{l}\text { p- } \\
\text { value* }\end{array}$ \\
\hline Mariara et al[18] & 2017 & 80 & 43 years & $51.6(42.2,65.6)$ & 0.007 \\
$\begin{array}{l}\text { Spies et al[28] } \\
2010\end{array}$ & 274 & 43 years & $40.8(22.1)$ & $<0.001$ \\
$\begin{array}{l}\text { Goodwin et } \\
\text { al[19] }\end{array}$ & 2008 & 2112 & 43 years & $46(29,65)$ & $<0.001$
\end{tabular}

214

215 *One sample Wilcoxon Signed rank test 


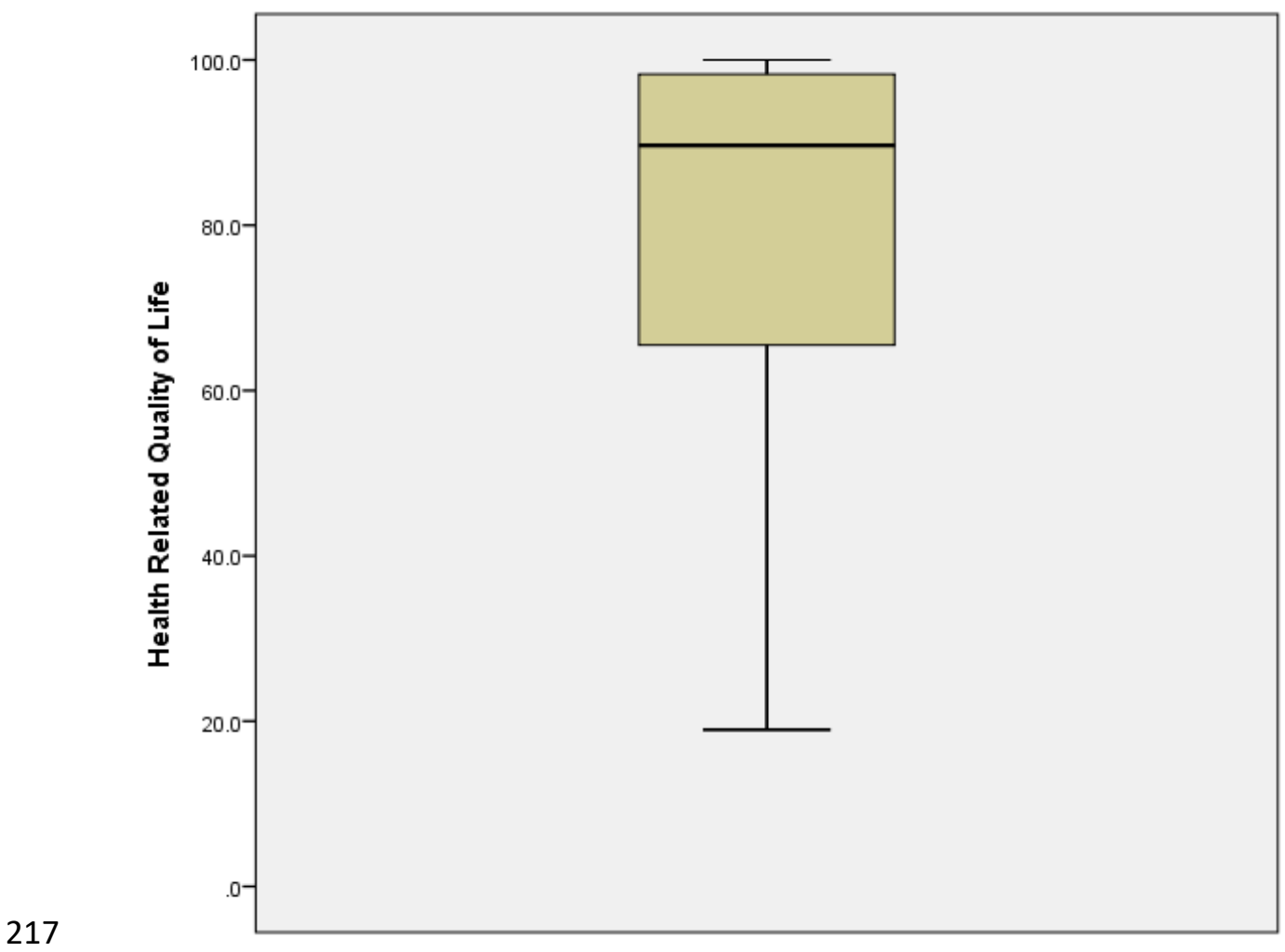

218 Figure 2: Participants Health Related Quality of Life Score

219 


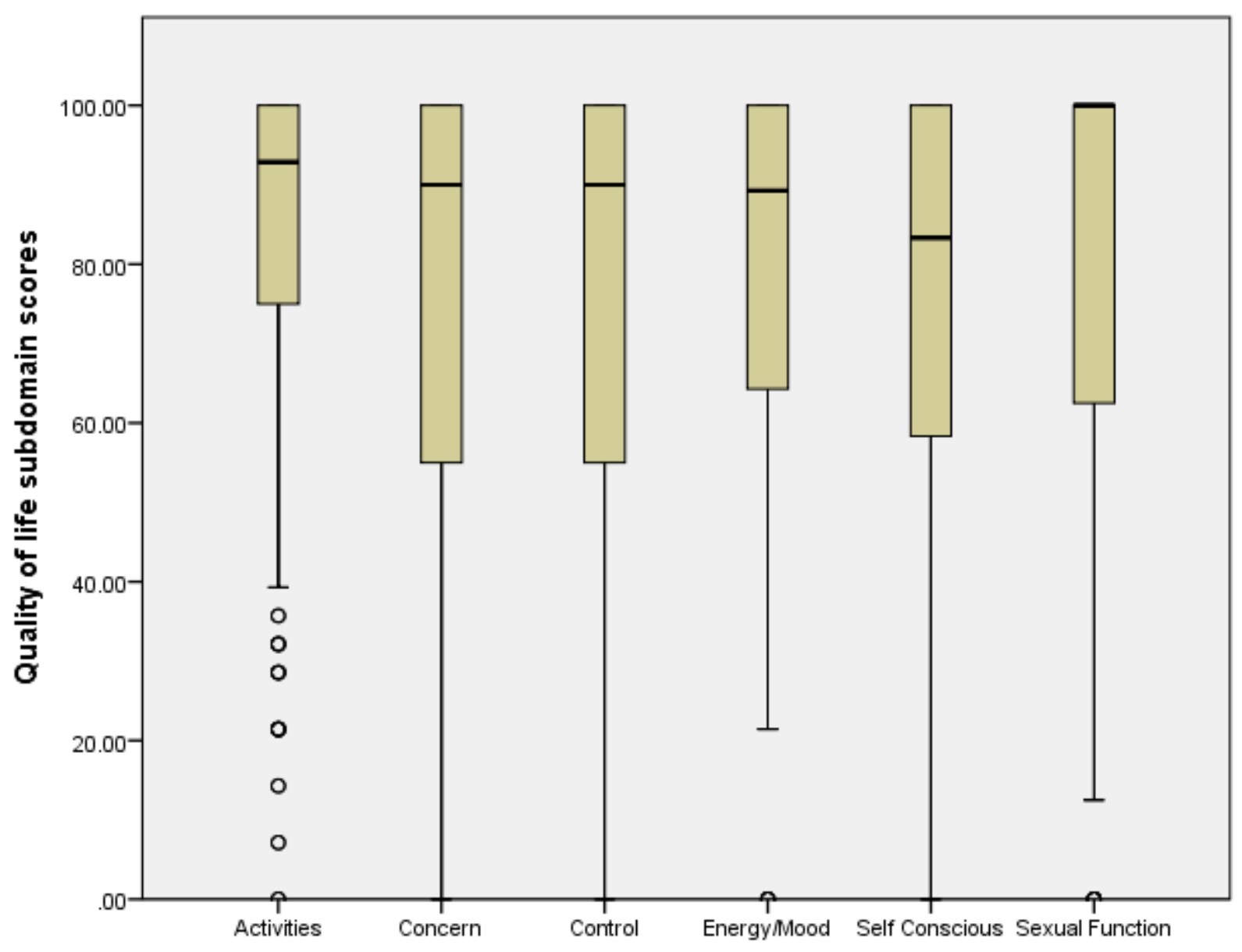

221 Figure 3: Long-term health related quality of life domain scores among women who had UFE

224 Age, baseline symptom score, uterine volume, dominant fibroid volume, number of years after 225 UFE procedure and current ongoing symptoms were evaluated as factors associated with the 226 quality of life following UFE. There was a strong negative correlation between symptom severity 227 and quality of life score (standard beta $=-0.498$, p-value $<0.001$ ) and weak negative correlation 228 with age (standard beta $=-0.23, \mathrm{p}$-value 0.044 ) as indicated in table 5 below. In this linear 229 regression model, for every unit increase in symptom severity score, the HRQOL score decreased 230 by 0.561 ( $p$ value <0.001). This inverse relationship is clearly visualized on the scatter plot 231 diagram on figure 4. No other statistically significant correlations were discovered in this analysis. 
233 Table 5: Linear regression results: Factors associated with quality of life

\begin{tabular}{|c|c|c|c|}
\hline Variable & Standard Beta & $\mathbf{9 5 \%}$ Confidence Interval & p-value \\
\hline Age & -0.230 & $-2.466--0.035$ & 0.044 \\
\hline Years after UFE & 0.099 & $-1.981-5.010$ & 0.391 \\
\hline Ongoing symptoms & -0.498 & $-0.786-0.336$ & $<0.001$ \\
\hline Baseline Uterine Volume & 0.097 & $-0.008-0.017$ & 0.493 \\
\hline Baseline Dominant Fibroid Volume & 0.131 & $-0.020-0.054$ & 0.354 \\
\hline $\begin{array}{l}\text { Baseline Transformed symptom } \\
\text { score }\end{array}$ & -0.235 & $-0.732-0.058$ & 0.093 \\
\hline
\end{tabular}

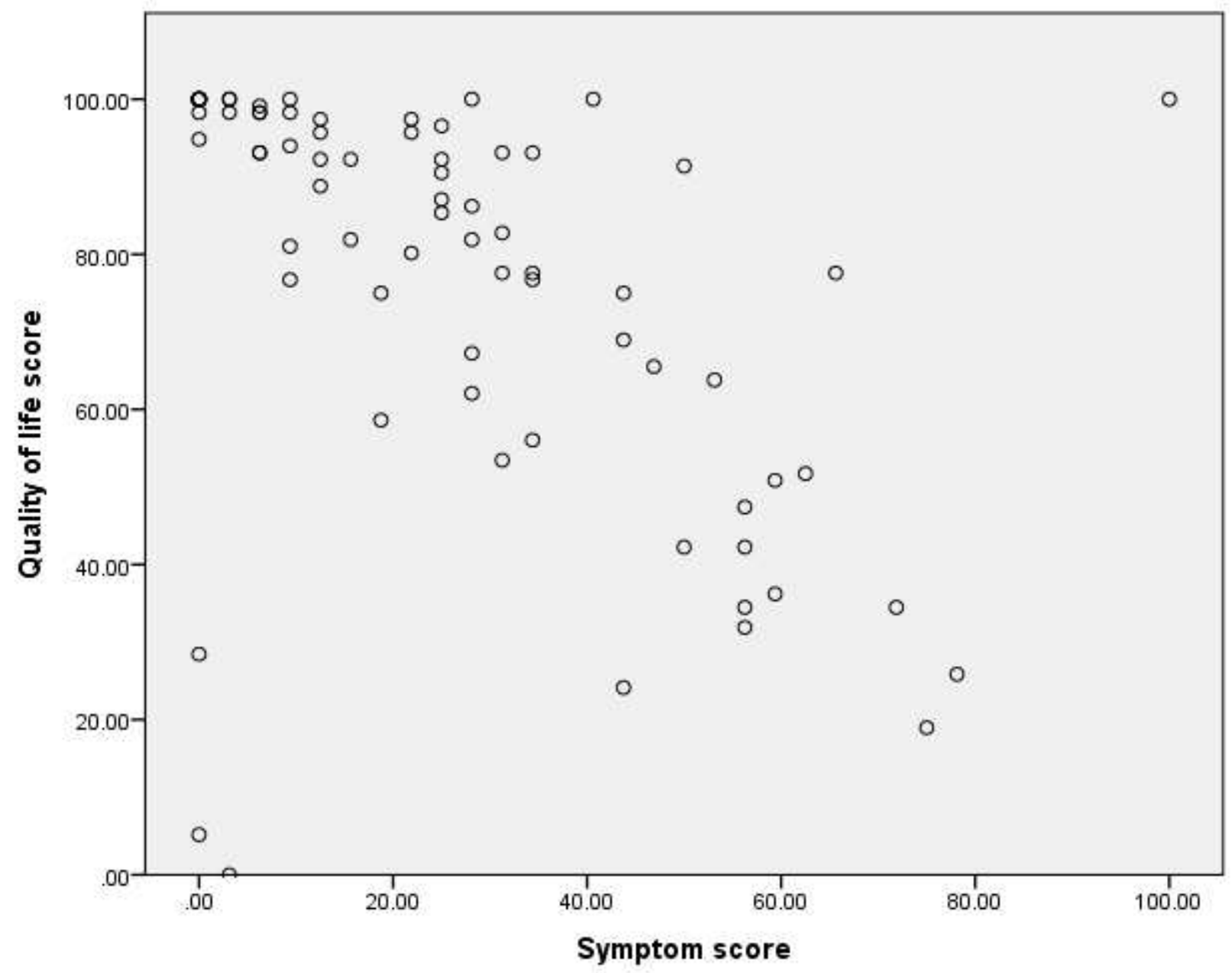


239 Baseline symptom severity data for 52 participants was available for comparison. The median symptom severity among the participants decreased from a baseline score of $54.7(43.8-65.6)$ to $21.9(6.3-42.2)$ as shown in figure 5 below. This decrease was statistically significant using the

242 related samples Wilcoxon signed rank test ( $p$ value $<0.001)$.

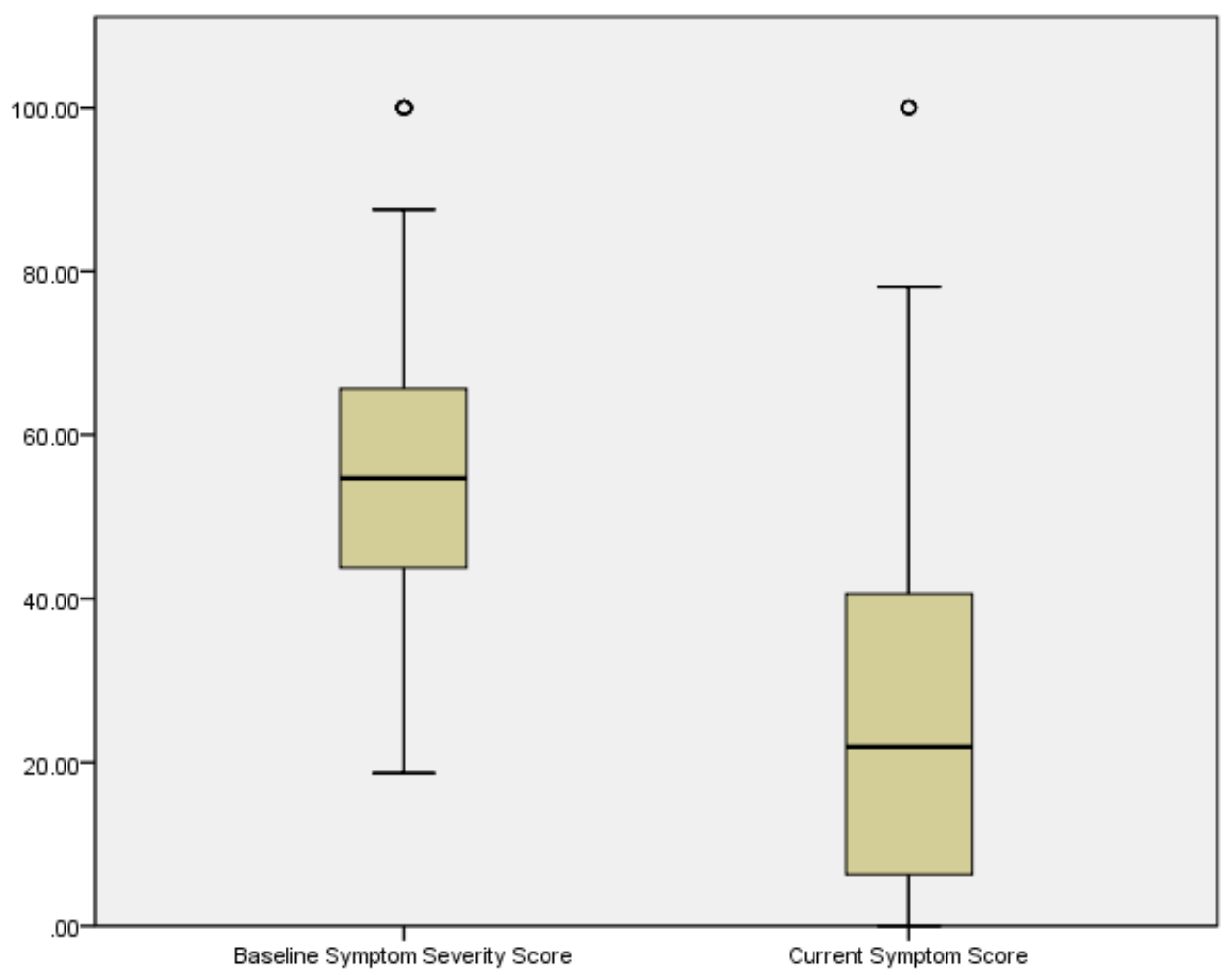

Figure 5: Change in participants symptom severity score from baseline

Age, baseline symptom score, uterine volume, dominant fibroid volume, number of years after UFE procedure and current health related quality of life were evaluated as factors associated with symptom severity. The only significant association for symptom severity was current health related quality of life following linear regression modelling as shown in table 6 below. This inverse relationship between symptom severity has already been reviewed in the quality of life results. 
253 Table 6: Linear regression results: Factors associated with symptom severity

\begin{tabular}{llll} 
Variable & Standard Beta & $\mathbf{9 5 \%}$ Confidence Interval & p-value \\
\hline Age & -0.006 & $-1.139-1.077$ & 0.956 \\
Years after UFE & -0.008 & $-3.223-3.012$ & 0.946 \\
Current quality of life & -0.498 & $-0.619--0.264$ & $<\mathbf{0 . 0 0 1}$ \\
Baseline Uterine Volume & -0.080 & $-0.012-0.007$ & 0.571 \\
Baseline Dominant Fibroid Volume & -0.260 & $-0.053-0.001$ & 0.063 \\
Baseline Transformed symptom score & 0.048 & $-0.267-0.376$ & 0.735 \\
\hline
\end{tabular}

\section{DISCUSSION}

255 We evaluated the fibroid related post UFE quality of life and symptom severity of 77 women with 256 a median age of 43 at baseline with a median duration of follow of 8 years. The 8-year quality of 257 life scores remained high and were statistically and clinically significantly better compared to 258 baseline scores before UFE in similar studies. The symptom severity scores were low compared 259 to baseline scores indicating that the benefits of UFE are persistent in the long term follow up.

260 Various studies have evaluated the quality of life among patients managed for uterine fibroids 261 with varying durations of follow up of between 1 to 3 years $[18,19,21]$. The results from these 262 studies indicate that UFE is an effective method of management of patients with fibroids. These 263 studies have evaluated the efficacy of UFE among participants recruited from multiple centers 264 across countries in Europe and the United States. The population structure and well-developed 265 highly funded healthcare settings sharply contrast healthcare systems in sub-Saharan Africa. 266 Hence, these study results cannot be generalized to a predominantly black population in a low267 income sub-Saharan country with complex challenges in health care provision. Our study setting 268 provides an insight into the long-term efficacy of UFE among patients with fibroids in this complex 269 health care setting. While sub-Saharan countries are still battling communicable diseases, a sharp 270 increase in non-communicable diseases such as diabetes, hypertension and cancer have put 271 additional strain in an already overstretched poorly funded healthcare. Additional challenges that 272 affect of management of symptomatic fibroids include difficulties in provision of surgical services 273 and blood transfusion. Our study results indicate that the long-term quality of life and symptom 274 severity is comparable to results in other studies done in high-income countries hence UFE could 
275 present an effective, minimally invasive, low risk alternative to surgery in management of 276 symptomatic uterine fibroids in this setting.

277 Our study has several strengths. Uterine fibroid embolization is a relatively new technology in 278 Africa with few studies documenting its use. The studies identified on UFE have studied other 279 UFE aspects such as radiological features and response of fibroids after UFE and short-term 280 quality of life changes for up to a year [18, 29]. Our study has sought to document the long-term 281 quality of life following UFE in a predominantly black population in sub-Saharan Africa. With the 282 immense untapped potential of UFE for management of symptomatic uterine fibroids, the long283 term quality of life is an important consideration among patients. We sought to address this gap 284 by conducting this study to contribute to the body of knowledge on this intervention in this part 285 of the world where fibroids are significant burden to health care with significant morbidity. By 286 conducting this research, we hope to spur interest in further interest in this field of research.

In depth data analysis revealed also various clinically important and relevant aspects of UFE such as repeat interventions following the UFE procedure due to non-resolution or recurrence of symptoms, fertility rates and patient satisfaction. The low reintervention rates at $14.3 \%$ (11 participants) was comparable to results in other studies [30]. Though our study was not powered to evaluate this aspect of UFE, the low reintervention rate is a reassuring trend. Other studies evaluating this aspect of UFE have documented high reintervention rates of between $25-30 \%$. The differences in various studies could be due to different definitions and duration of follow up to determine what constitutes a reintervention. In the EMMY trial and subsequent follow up studies, reintervention constituted only hysterectomies following UFE. Of note, the patients in this trial and follow studies were follow up for up to 10 years [25, 31]. In the systematic review by Sandberg et al, the duration of follow up was up to 5 years [30]. This significant heterogeneity in the studies have yielded mixed results. A prospective comparative study with life tables and 304 survival analysis in our local setting would yield important data and give a true picture of the 305 situation. 
Several limitations were identified in this study. Some of the data collected retrospectively such as baseline scores and other data elements was either incomplete or entirely missing. Baseline quality of life data was completely missing while data for symptom severity scores was only available for 52/77 participants. The retrospective nature of data collection was informed by relative low numbers of UFE interventions per year hence making recruitment of prospective participants a non-viable option due time constraints. To address this limitation, we compared our study results with baseline and post intervention scores in similar studies utilizing the same data collection tool $[19,21]$. We recommend a prospective cohort study for future studies in this field to yield rich data.

Whereas the online data collection process improved responses from different participants without being limited by geographical spread and significantly reducing the cost of data collection, it had several challenges. Some participants would have been compelled to fill by just clicking on the options provided. This could have been the case where some respondents indicated significant ongoing symptoms whereas they reported high quality of life. These seemingly discrepant responses were flagged in four participants during the data cleaning and analysis process. It is also possible they could have misunderstood the questionnaire. Although assistance was available for clarification via email or telephone contacts, few respondents contacted the investigators for clarification. Future data collection processes could employ real time assistance during the online data collection process to improve the quality of data collected. A mixed method approach would also seek to clarify the responses obtained by giving a qualitative perspective.

We tried to minimize several biases inherent to our study design. As the participants were being followed up from the same center that carried out the procedure, they have been biased to respond favorably or unfavorably depending of their experience hence leading to skewing of the data distribution. We minimized this bias by reassuring the participants the study responses were confidential. No physicians involved in the initial care of the patients were involved in the data collection process hence minimizing this desirability bias.

\section{CONCLUSION}

UFE results in clinically sustained improvement in health related quality of life and symptom control among patients with uterine fibroids. The effect of UFE on fertility is unclear with a trend towards increased rate of miscarriage and reduced pregnancy rates. UFE presents a safe, efficacious minimally invasive, uterine sparing alternative in the management of uterine fibroids 
337 especially in low resource settings where transfusion of blood and blood products might be 338 challenging. Clinicians should provide adequate counselling to patients concerning the efficacy 339 and expected follow up after UFE.

340 LIST OF ABBREVIATIONS

341 AKUHN

342

343

344

345

346

347

348

349

350

351

352

353

354

355

356

357

358

359

360

361
Aga Khan University Hospital, Nairobi

Anti - Mullerian Hormone

High Intensity Focused Ultrasound

Health Related Quality of Life

International Business Machine Statistical Product and

Service Solutions

Internet Protocol

Inter Quartile Range

Short Form -36 Questionnaire

Symptom Severity Score

Uterine Fibroid Embolization

Uterine Fibroid Symptom and Quality of Life

Questionnaire

\section{DECLARATIONS}

\section{ETHICS AND CONSENT TO PARTICIPATE}

The study received ethical clearance from Aga Khan University Ethics Review Committee (Ref: 2019/REC-19 (ver-3)). Informed consent was obtained from all study participants. The study was conducted in accordance to the relevant guidelines and regulations.

\section{CONSENT FOR PUBLICATION}

\section{Not Applicable}


363 The data collected and analysed in this project is available on request via the email 364 mickiks@gmail.com. The available data is devoid of any information that may potentially breach 365 the confidentiality of study participants.

366 COMPETING INTERESTS

367 The authors declare that they have no competing interests.

\section{CONFLICT OF INTEREST}

369 There are no conflicts of interest to declare.

\section{FUNDING}

371 The study was funded by Aga Khan University. The funder had no role in the design, data 372 collection and analysis of the results.

AUTHORS' CONTRIBUTIONS

MK Conceptualisation, data collection, data analysis, writing of the manuscript.

MT Overall guidance, content development and supervision of the project to completion

TO Supervision, Content development, participant recruitment and data collection process

AL Supervision and revision of the drafts, methodology guidance and preparation of the manuscript publication process

\section{ACKNOWLEDGEMENT}

We acknowledge all the study participants for their willingness and involvement in this study. Their participation was invaluable and critical to the completion of this project.

I am appreciative of the services of James Orwa who assisted statistical analysis of the study.

I acknowledge my supervisors, family, colleagues and friends who encouraged and cheered me on this project.

\section{REFERENCES}

1. Cardozo, E.R., et al., The estimated annual cost of uterine leiomyomata in the United States. Am J Obstet Gynecol, 2012. 206(3): p. 211 e1-9.

2. Baird, D.D., et al., High cumulative incidence of uterine leiomyoma in black and white women: ultrasound evidence. Am J Obstet Gynecol, 2003. 188(1): p. 100-7.

3. Global Burden of Disease Study, C., Global, regional, and national incidence, prevalence, and years lived with disability for 301 acute and chronic diseases and injuries in 188 countries, 1990-2013: a systematic analysis for the Global Burden of Disease Study 2013. Lancet, 2015. 386(9995): p. 743-800. 
4. Downes, E., et al., The burden of uterine fibroids in five European countries. Eur J Obstet Gynecol Reprod Biol, 2010. 152(1): p. 96-102.

5. Marshall, L.M., et al., Variation in the incidence of uterine leiomyoma among premenopausal women by age and race. Obstet Gynecol, 1997. 90(6): p. 967-73.

6. Marsh, E.E., et al., Racial differences in fibroid prevalence and ultrasound findings in asymptomatic young women (18-30 years old): a pilot study. Fertil Steril, 2013. 99(7): p. 1951-7.

7. Templeman, C., et al., Risk factors for surgically removed fibroids in a large cohort of teachers. Fertil Steril, 2009. 92(4): p. 1436-46.

8. Huyck, K.L., et al., The impact of race as a risk factor for symptom severity and age at diagnosis of uterine leiomyomata among affected sisters. Am J Obstet Gynecol, 2008. 198(2): p. 168 e1-9.

9. Kjerulff, K.H., et al., Uterine leiomyomas. Racial differences in severity, symptoms and age at diagnosis. J Reprod Med, 1996. 41(7): p. 483-90.

10. Moore, A.B., et al., Association of race, age and body mass index with gross pathology of uterine fibroids. J Reprod Med, 2008. 53(2): p. 90-6.

11. Peddada, S.D., et al., Growth of uterine leiomyomata among premenopausal black and white women. Proc Natl Acad Sci U S A, 2008. 105(50): p. 19887-92.

12. Myers, E.R., et al., Prospective data collection of a new procedure by a specialty society: the FIBROID registry. Obstet Gynecol, 2005. 106(1): p. 44-51.

13. Borah, B.J., et al., The impact of uterine leiomyomas: a national survey of affected women. Am J Obstet Gynecol, 2013. 209(4): p. 319 e1-319 e20.

14. Goodwin, S.C., et al., Preliminary experience with uterine artery embolization for uterine fibroids. J Vasc Interv Radiol, 1997. 8(4): p. 517-26.

15. Spies, J.B., et al., The FIBROID Registry: symptom and quality-of-life status 1 year after therapy. Obstet Gynecol, 2005. 106(6): p. 1309-18.

16. Lohle, P.N., et al., Long-term outcome of uterine artery embolization for symptomatic uterine leiomyomas. J Vasc Interv Radiol, 2008. 19(3): p. 319-26.

17. Hirst, A., et al., A multi-centre retrospective cohort study comparing the efficacy, safety and cost-effectiveness of hysterectomy and uterine artery embolisation for the treatment of symptomatic uterine fibroids. The HOPEFUL study. Health Technol Assess, 2008. 12(5): p. 1-248, iii.

18. Mariara, C., et al., One year symptom severity and health-related quality of life changes among Black African patients undergoing uterine fibroid embolisation. BMC Res Notes, 2017. 10(1): p. 240.

19. Goodwin, S.C., et al., Uterine artery embolization for treatment of leiomyomata: long-term outcomes from the FIBROID Registry. Obstet Gynecol, 2008. 111(1): p. 22-33.

20. Pinto, I., et al., Uterine fibroids: uterine artery embolization versus abdominal hysterectomy for treatment--a prospective, randomized, and controlled clinical trial. Radiology, 2003. 226(2): p. 425-31.

21. Spies, J.B., et al., Long-term outcome of uterine artery embolization of leiomyomata. Obstet Gynecol, 2005. 106(5 Pt 1): p. 933-9.

22. Gabriel-Cox, K., et al., Predictors of hysterectomy after uterine artery embolization for leiomyoma. Am J Obstet Gynecol, 2007. 196(6): p. 588 e1-6.

23. Popovic, M., et al., Long-term quality of life assessment among patients undergoing uterine fibroid embolization. AJR Am J Roentgenol, 2009. 193(1): p. 267-71. 
440 24. Moss, J.G., et al., Randomised comparison of uterine artery embolisation (UAE) with surgical treatment in patients with symptomatic uterine fibroids (REST trial): 5-year results. BJOG, 2011. 118(8): p. 936-44.

25. van der Kooij, S.M., et al., Uterine artery embolization vs hysterectomy in the treatment of symptomatic uterine fibroids: 5-year outcome from the randomized EMMY trial. Am J Obstet Gynecol, 2010. 203(2): p. 105 e1-13.

26. Spies, J.B., et al., The UFS-QOL, a new disease-specific symptom and health-related quality of life questionnaire for leiomyomata. Obstet Gynecol, 2002. 99(2): p. 290-300.

27. Jinwoo Park, M.J., A Note on Determination of Sample Size for a Likert Scale. Communications of the Korean Statistical Society, 2009. 16(4): p. 669-773.

28. Spies, J.B., et al., Outcomes from leiomyoma therapies: comparison with normal controls. Obstet Gynecol, 2010. 116(3): p. 641-52.

29. Mutai, J.K., et al., Uterine fibroid embolization for symptomatic fibroids: study at a teaching hospital in kenya. J Clin Imaging Sci, 2015. 5: p. 18.

30. Sandberg, E.M., et al., Reintervention risk and quality of life outcomes after uterinesparing interventions for fibroids: a systematic review and meta-analysis. Fertil Steril, 2018. 109(4): p. 698-707 e1.

31. de Bruijn, A.M., et al., Uterine artery embolization vs hysterectomy in the treatment of symptomatic uterine fibroids: 10-year outcomes from the randomized EMMY trial. Am J Obstet Gynecol, 2016. 215(6): p. 745 e1-745 e12. 
Figures

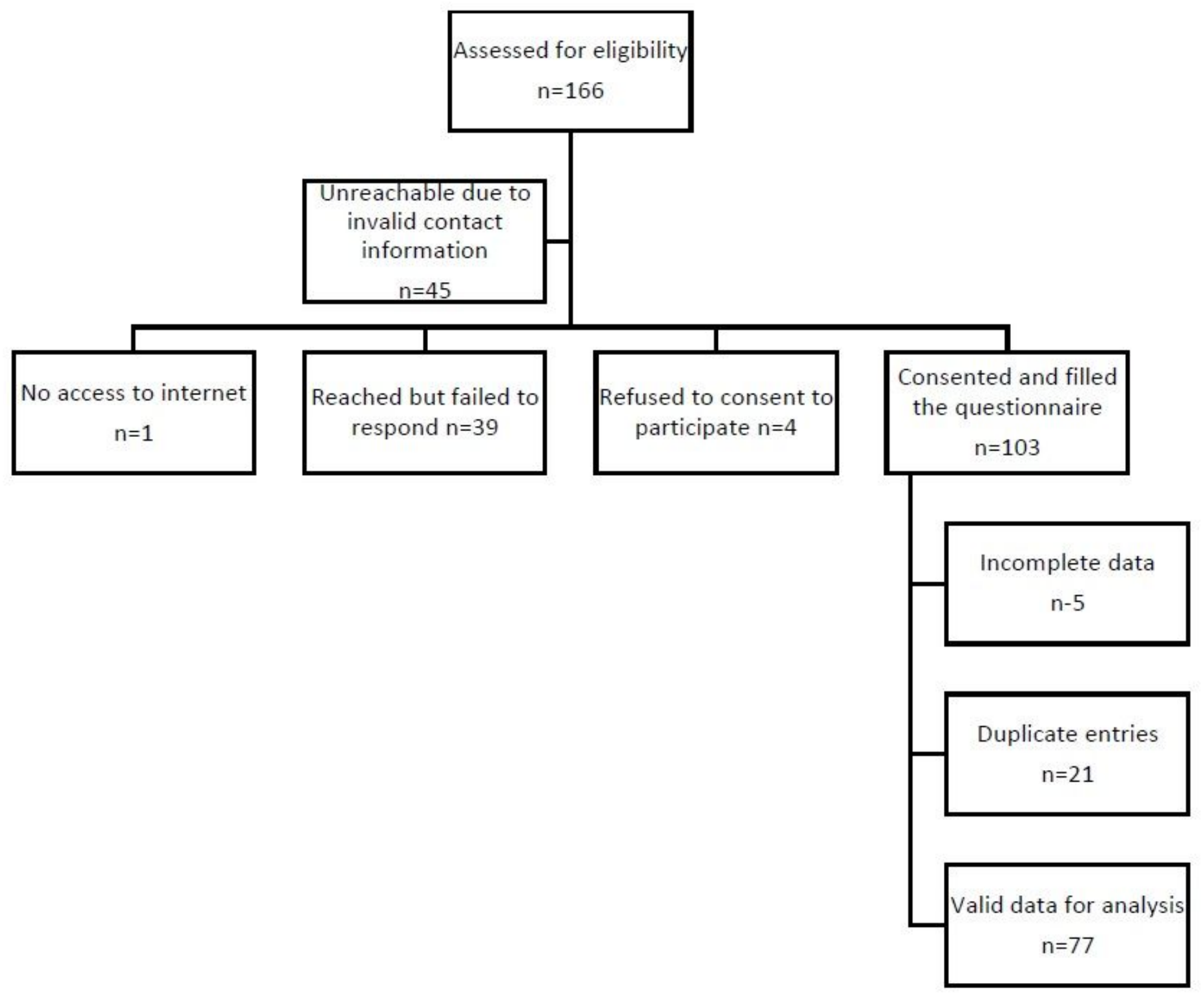

Figure 1

Participant enrollment flow to the UFE Study 


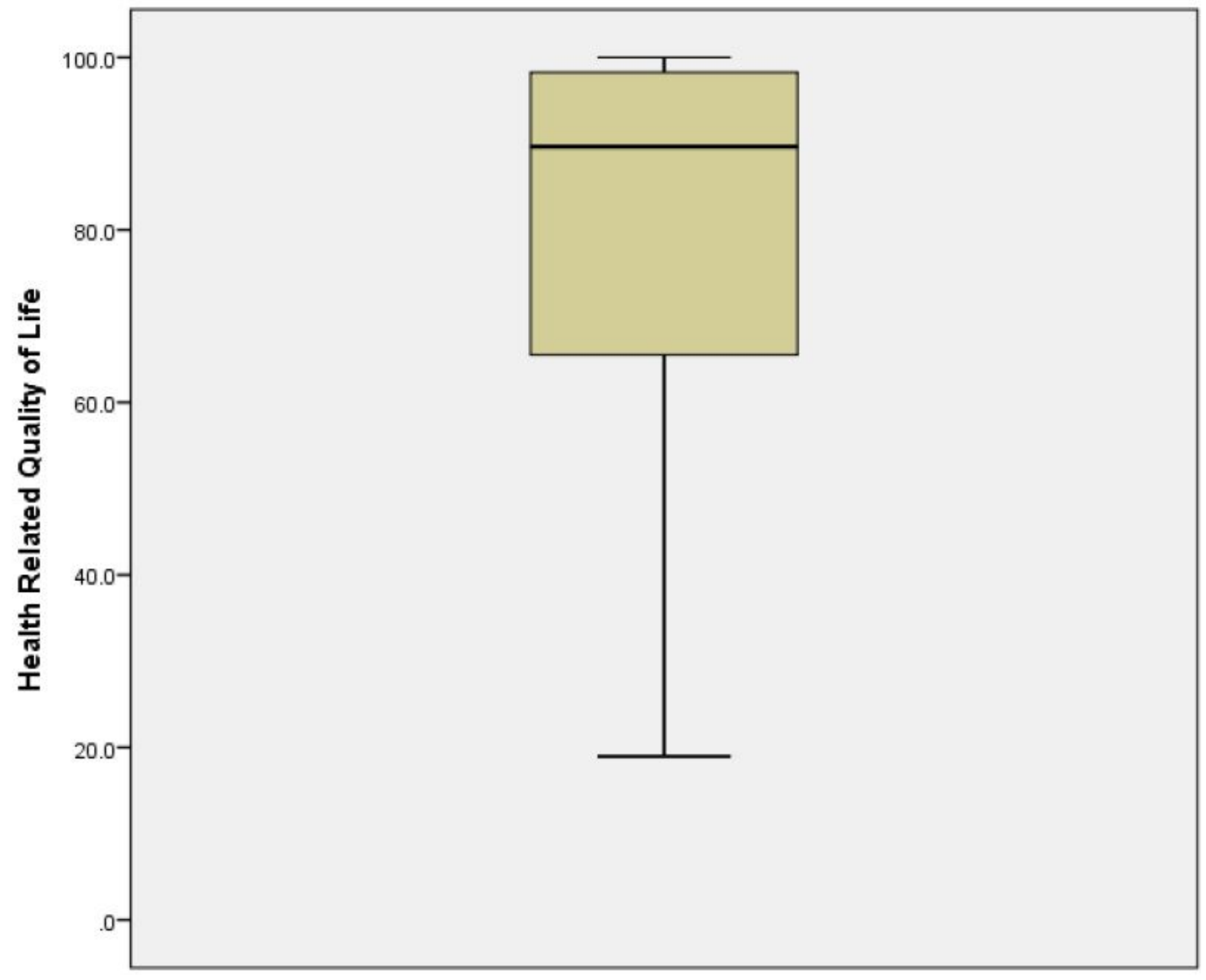

Figure 2

Participants Health Related Quality of Life Score 


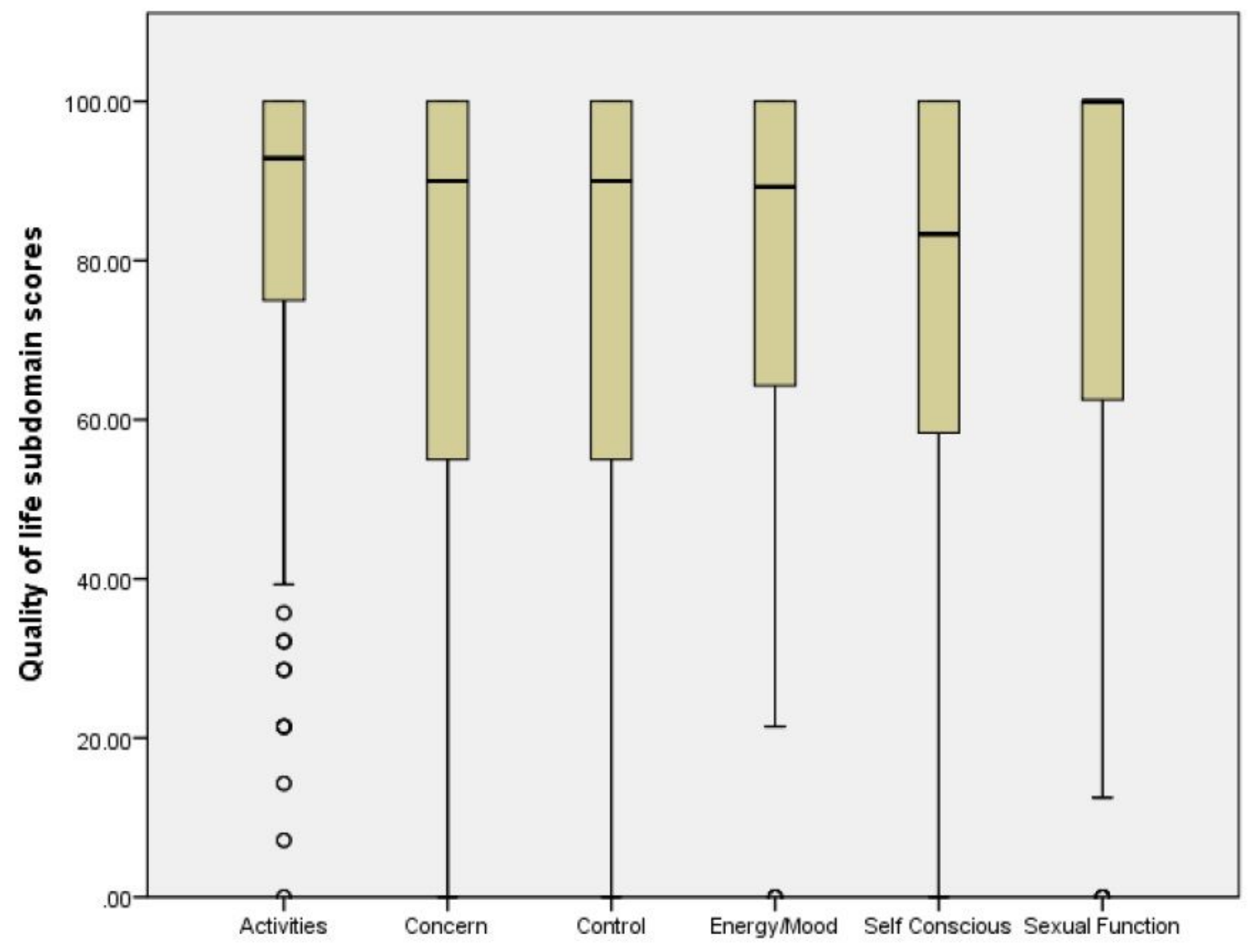

Figure 3

Long-term health related quality of life domain scores among women who had UFE 


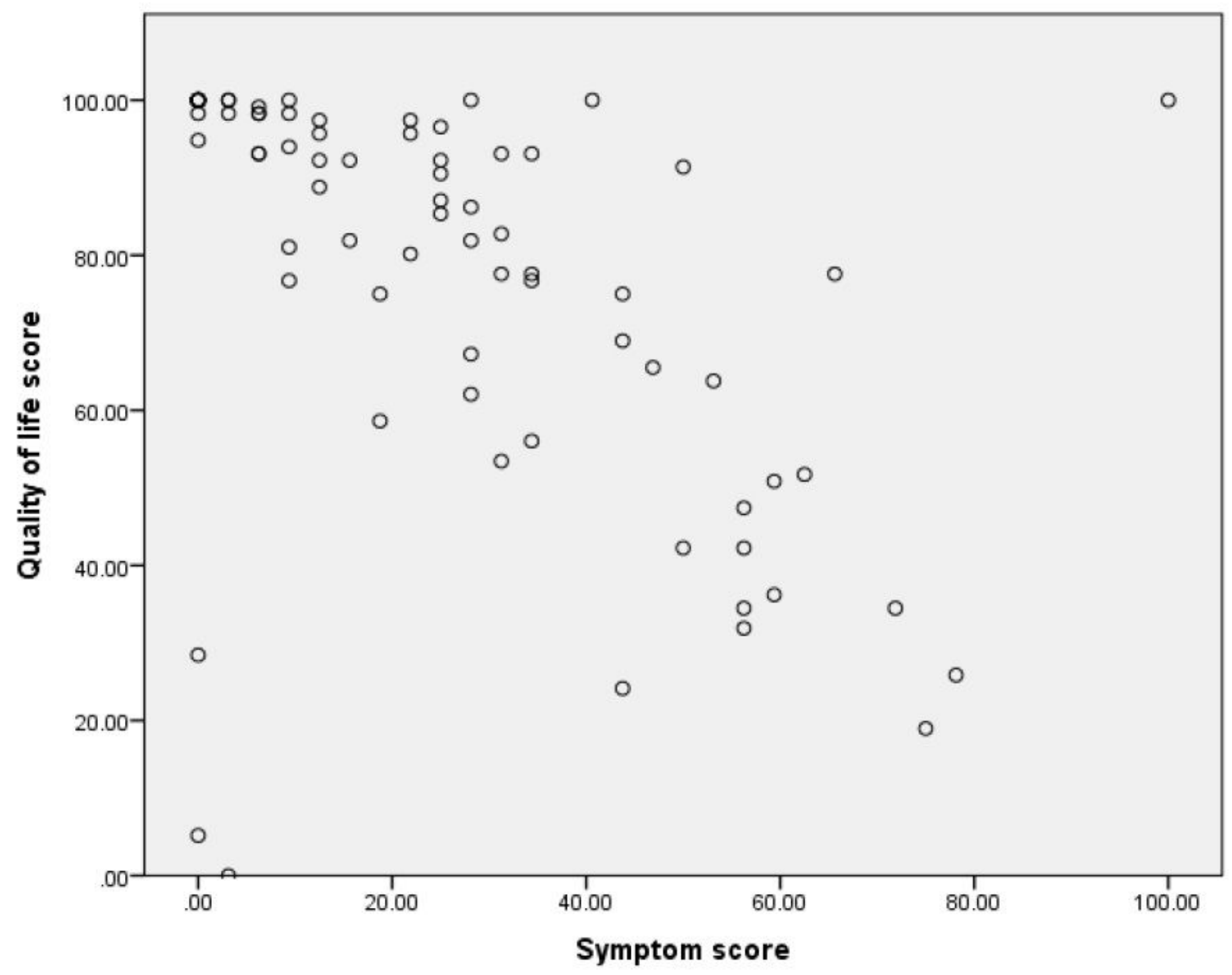

Figure 4

Inverse relationship between quality of life and symptom severity scores 


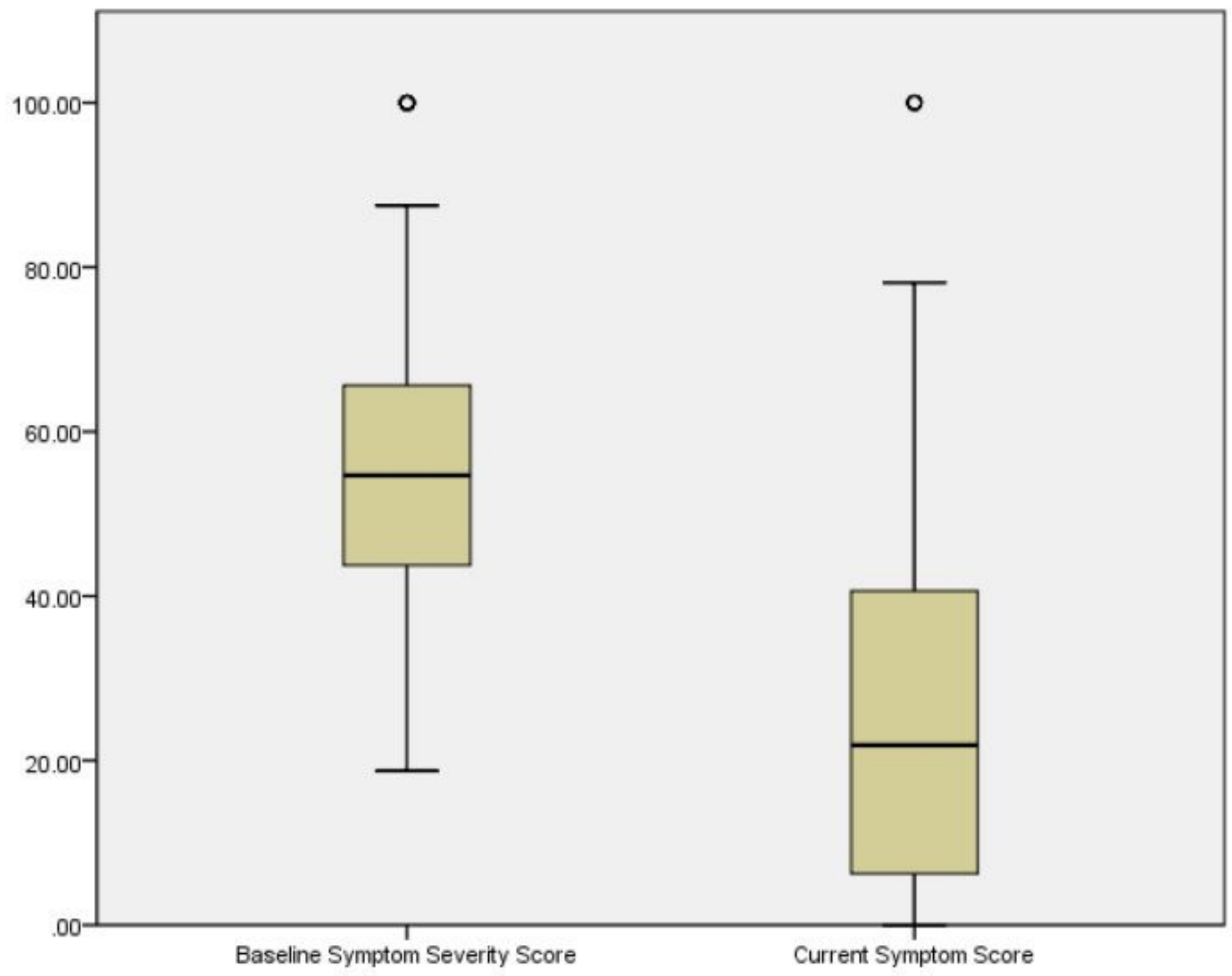

Figure 5

Change in participants symptom severity score from baseline 